Elspeth M. McLachlan

\title{
Diversity of sympathetic vasoconstrictor pathways and their plasticity after spinal cord injury
}

Received: 21 December 2006

Accepted: 2 January 2007

Published online: 30 January 2007
Prof. E.M. McLachlan ( $\square)$

Spinal Injuries Research Centre

Prince of Wales Medical Research Institute

Gate 1 Barker Street

Randwick (NSW) 2031, Australia

Tel.: +61-2/9399-1031

Fax: +61-2/9399-1034

E-Mail: e.mclachlan@unsw.edu.au
Abstract Sympathetic vasoconstrictor pathways pass through paravertebral ganglia carrying ongoing and reflex activity arising within the central nervous system to their vascular targets. The pattern of reflex activity is selective for particular vascular beds and appropriate for the physiological outcome (vasoconstriction or vasodilation). The preganglionic signals are distributed to most postganglionic neurones in ganglia via synapses that are always suprathreshold for action potential initiation (like skeletal neuromuscular junctions).

Most postganglionic neurones receive only one of these "strong" inputs, other preganglionic connections being ineffective. Preand postganglionic neurones discharge normally at frequencies of $0.5-1 \mathrm{~Hz}$ and maximally in short bursts at $<10 \mathrm{~Hz}$. Animal experiments have revealed unexpected changes in these pathways following spinal cord injury. (1) After destruction of preganglionic neurones or axons, surviving terminals in ganglia sprout and rapidly re-establish strong connections, probably even to inappropriate postganglionic neurones. This could explain aberrant reflexes after spinal cord injury. (2) Cutaneous (tail) and splanchnic (mesenteric) arteries taken from below a spinal transection show dramatically enhanced responses in vitro to norepinephrine released from perivascular nerves. However the mechanisms that are modified differ between the two vessels, being mostly postjunctional in the tail artery and mostly prejunctional in the mesenteric artery. The changes are mimicked when postganglionic neurones are silenced by removal of their preganglionic input. Whether or not other arteries are also hyperresponsive to reflex activation, these observations suggest that the greatest contribution to raised peripheral resistance in autonomic dysreflexia follows the modifications of neurovascular transmission.

Key words sympathetic nervous system - sympathetic ganglia . autonomic dysreflexia . vasoconstriction - norepinephrine $\cdot$ autonomic nervous system 


\section{Introduction}

The sympathetic nervous system supplies blood vessels throughout the body with vasoconstrictor axons that control local blood flow and contribute to total peripheral resistance. Signals arising in the central nervous system send specific controls to different tissues according to the functional response for each organ by means of reflexes and patterned regulation from higher centres [14]. The sympathetic outflow arises in the thoracolumbar cord, where it is particularly vulnerable to spinal cord injuries, being damaged directly if the lesion involves regions between T1 and L3 or the ventral roots at these levels, and being disconnected from the higher control centres by injuries to descending pathways in the cervical cord. The degree of disordered visceral and vascular function that ensues depends on the level and the severity of trauma in each individual.

The great majority of all sympathetic preganglionic neurones are vasoconstrictor in function and make synapses in ganglia of the paravertebral chain from which they project through grey rami and spinal nerves to the vasculature of the head, neck, trunk and limbs. Because the sympathetic outflow is restricted to thoracolumbar segments, preganglionic neurones in the upper thoracic segments control vessels in the head and neck and those in the lowest thoracic and upper lumbar segments control vessels in the pelvic organs and hindlimbs. Thus, for example, postganglionic neurones in the superior cervical ganglia receive inputs from T1-4 segments [25] whereas inputs to neurones in the lower lumbar paravertebral chain projecting to the hindlimbs mostly arise in the last two segments of the thoracolumbar sympathetic outflow [1]. Many visceral vasoconstrictor neurones also lie in the paravertebral chain and send their axons to the viscera via the various splanchnic nerves. The properties of these pathways supplying the vasculature and their modification after spinal cord injury (SCI) are the main topics of this review.

\section{Patterns of natural activity in sympathetic vasoconstrictor pathways}

Impulses travelling in sympathetic vasoconstrictor pathways arise in preganglionic neurones by integration of excitatory and inhibitory signals that arise supraspinally, intersegmentally, and segmentally. In vasoconstrictor pathways, the patterns of ongoing or tonic firing of preganglionic neurones originate in the rostral ventrolateral medulla [3]. However many descending excitatory glutamatergic axons and inhibitory axons containing gamma-amino-butyric acid and/or glycine that arise both locally and sup- raspinally impinge on preganglionic neurones [17]. These inputs may be modulated by amines and peptides co-released from these pathways [26]. The preganglionic neurones integrate reflex signals from e.g. arterial baroreceptor and chemoreceptors via the brainstem, cutaneous and visceral nociceptors at spinal levels, signals arising from higher levels of the CNS, etc. Strong reciprocal links between vasomotor and respiratory centres in the brainstem, together with blood pressure swings related to breathing, mean that vasoconstrictor activity tends to be linked to the respiratory cycle as well as the cardiac cycle $[8,10,19$, 32].

The final output frequency and pattern of discharge in individual sympathetic axons is modulated by the membrane properties of the preganglionic neurones. Action potentials in these neurones are triggered by the summed depolarization produced by many small excitatory inputs $[5,12,29]$ combined with inhibitory synaptic potentials arising from both local spinal interneurones and direct descending inhibitory pathways $[6,7]$. The frequency of preganglionic discharge is limited to short bursts at $<10 \mathrm{~Hz}$ by a large $\mathrm{Ca}^{2+}$-activated $\mathrm{K}^{+}$conductance (and ensuing afterhyperpolarization) that follows each action potential, together with a prolonged transient (A-type) $\mathrm{K}^{+}$current $[4,29]$. The average ongoing preganglionic discharge in vasoconstrictor pathways in anaesthetized animals is $0.5-1 \mathrm{~Hz}$ [14].

The natural activity of individual postganglionic neurones projecting to different target tissues has been described by recording extracellularly spontaneous and evoked signals from sympathetic axons in peripheral nerves projecting to a known target tissue $[14,35]$. Ongoing activity of individual units is recognized by the spike configuration and the unit functionally characterized by its responses to natural stimuli. Physiologically relevant responses are recorded from e.g. presumed sudomotor axons in cutaneous nerves immediately preceding secretion from sweat glands. Perhaps surprisingly, when simultaneous recordings are made from skin and muscle vasoconstrictor axons, the responses are generally opposite. For example, baroreflex inhibition is powerful in muscle vasoconstrictor (MVC) axons but weak or absent in cutaneous vasoconstrictor (CVC) axons. Chemoreceptor activation stimulates MVCs but inhibits CVCs, nociceptor stimuli excite CVCs but inhibit MVCs [13, 14]. These differences reflect what is known about blood flow through skin and muscle, with the former involved primarily in temperature regulation and the latter in regulation of peripheral resistance at rest. Similar differences in skin and muscle vasoconstrictor axons are observed in microneurographic recordings from conscious humans in which the excitatory effects of emotion on 
CVCs and mental stress on MVCs are revealed [33, 34]. The importance of these observations is that they make the concept of a generalized sympathetic vasoconstrictor "tone" inappropriate.

When this could be studied, the patterns and frequencies of preganglionic discharge have convincingly parallelled those of the postganglionic projections in the same pathway. Thus the average postganglionic discharge frequency in vasoconstrictor pathways is $0.5-1 \mathrm{~Hz}[13,14]$.

\section{Transmission of vasoconstrictor signals through sympathetic ganglia}

The similarity between pre- and postganglionic discharge raises questions about the purpose of the synapses in sympathetic ganglia. Each preganglionic vasoconstrictor neurone branches to form synapses with multiple postganglionic neurones in several adjacent ganglia in the paravertebral chain. However only a subset of inputs (generally those that arise in one spinal segment) transmits the centrally derived command signal to the postganglionic neurones. This results because, in individual postganglionic neurones, transmission occurs in an all-or-none manner from a single input, as at the skeletal neuromuscular junction [21]. By recording intracellularly, and recruiting preganglionic inputs using graded stimuli, each postganglionic neurone can be shown to receive one "strong" excitatory input (rarely 2 or 3 ) that is always suprathreshold for action potential initiation, as well as several subthreshold excitatory inputs. (There are no inhibitory synapses in sympathetic ganglia.) As the safety factor for transmission at most ganglionic synapses is very high, high concentrations of nicotinic antagonists are necessary to block ganglionic transmission. The important role of the strong input has been confirmed in intracellular recordings in vivo $[22,30]$, which show that subthreshold inputs rarely summate, due to both the low preganglionic firing frequency and the large afterhyperpolarization after the action potential (again due to a $\mathrm{Ca}^{2+}$-activated $\mathrm{K}^{+}$conductance) triggered by the strong input. In practice, subthreshold inputs appear largely redundant. The total number of preganglionic inputs parallels the number of dendrites of the postganglionic neurone and these increase with animal size [27]. It is anticipated that each human paravertebral neurone receives at least 20 synaptic inputs, but that, in most cases, only one of these is functionally effective.

The connectivity described above means that ganglionic synapses in vasoconstrictor pathways simply relay the centrally-derived signals to the periphery without alteration. Thus the ganglia act primarily as distributors of the central pattern of impulses, each preganglionic axon activating many postganglionic neurones. Teleologically, this can be seen to reduce the need to provide large numbers of preganglionic neurones in the spinal cord to control the extensive vasculature throughout the body. However, although the preganglionic axons diverge to contact many ganglion cells ( $\sim 150$ in the rat, [28], the redundancy of many of these contacts means that the functional autonomic equivalent to the motor unit is much smaller (only $\sim 15$ in rat, perhaps $>100$ in humans).

\section{Changes in vasoconstrictor outflow after spinal cord injury}

In microneurographic recordings from skin and muscle nerves in conscious relaxed intact humans (Fig. 1A), recordings from sympathetic axons in muscle nerves show bursts of activity in rhythm with the arterial pulse wave that wax and wane with respiration [31], reflecting the cyclic inhibition of MVCs by baroreceptor input. Such bursts are rarely recorded in CVCs where ongoing activity is largely unsynchronized. During simultaneous recordings from skin and muscle fascicles, brief electrical stimulation of skin leads to a burst of activity in the CVCs but inhibition of ongoing bursts in MVCs. This reflects the reflex activation of CVCs and inhibition of MVCs by nociceptor stimuli.

Such paired recordings from leg nerves of people with thoracic spinal cord injury, showed completely different responses (Fig. 1B). The cardiac related bursts were absent and the signals from both nerves showed little background activity. Electrical stimuli to the skin produced identical brief bursts of activity in both skin and muscle nerves. These observations reflect the removal of both descending sympathetic drive with periodic baroreceptor inhibition and the conversion of the nociceptor-evoked reflex in MVCs from inhibitory to excitatory. This reversal of the

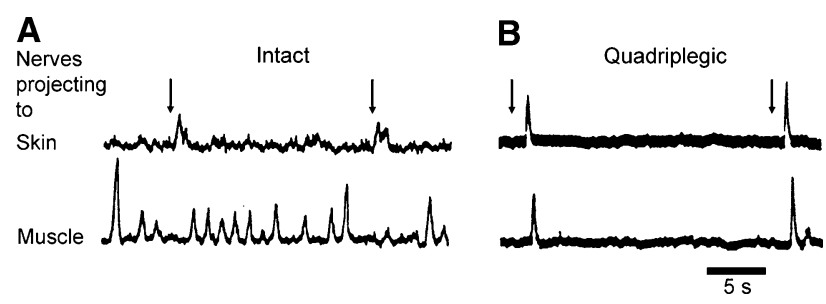

Fig. 1 Extracellular recording of multiunit sympathetic activity in skin and muscle nerves of conscious humans. (A) from intact individual; (B) from a subject with complete spinal cord transection at C6. At arrows, the skin over the forearm was stimulated electrically. Note the absence of ongoing activity in both nerves and the synchronous activation by the stimulus after spinal cord injury. Modified from Stjernberg et al. (1986) 
spinal nociceptor reflex has also been demonstrated in chronic spinal cats [15]. It should be noted that sympathoexcitation following nociceptor stimulation was transient without evidence of enhancement or prolongation of the reflex sympathetic discharge although the vasoconstriction was prolonged.

\section{Changes in connectivity in paravertebral ganglia after destruction of preganglionic neurones}

The consequences for the remaining sympathetic pathways of damage to preganglionic neurones or their axons by a spinal injury can be studied in experimental animals by partially denervating the paravertebral ganglia and observing the types of connection made by the remaining terminals. Extensive collateral sprouting of the remaining preganglionic axons occurs [16, 23]. Transection of the descending lumbar paravertebral chain just above the last segmental preganglionic outflow left postganglionic neurones in the next more caudal lumbar ganglion with $<1$ input, the great majority being subthreshold (Ireland 1999); the residual preganglionic input in the last sympathetic segment formed strong inputs on only $10 \%$ of the neurones. After 45 weeks, however, $70 \%$ of neurones in the same ganglion had a large strong input as well as $\sim 2$ subthreshold inputs. Thus. the remaining preganglionic neurones had sprouted and preferentially formed strong inputs, even on neurones that were completely denervated by the lesion. New strong inputs must have formed on some neurones with a peripheral function that differed from that of the original preganglionic input [16]. The formation of such aberrant strong inputs after SCI might explain more generalized vasoconstriction, or the activation of sudomotor neurones by reflexes normally directed at peripheral vessels.

\section{Autonomic dysreflexia}

While modified vascoconstrictor reflexes in spinallyinjured people can be mimicked in experimental animals, they do not predict the dramatic and lifethreatening rises in arterial blood pressure that are known as autonomic dysreflexia. These uncontrolled hypertensive episodes are usually triggered by overdistension of bladder or bowel but many also arise from nociceptor activation from a pressure sore or other unheeded injury below the spinal cord injury $[20,36]$. Such episodes are usually relieved by removal of the stimulus but, if the trigger is unknown, treatment with vasodilators requires hospitalisation which is inconvenient and expensive. Other symptoms of dysreflexia are excessive sweating and muscle spasms. Dysreflexia is typically seen only if the lesion is at or above T5-6.

It is generally believed that the unrestrained hypertension results from the absence of descending inhibitory control by baroreceptors and clearly the loss of this reflex control is highly relevant. The reason that T5 is critical as the cut-off for dysreflexic episodes is presumably because baroreflex vagal slowing of heart rate and inhibition of intact sympathetic pathways only to the head, neck and arm vasculature are not sufficient to buffer increases in vascular resistance below the injury. The simultaneous activation of both cutaneous and muscle vascular beds below the injury would contribute a large increase in peripheral resistance, and the effect would be more dramatic if the visceral vasculature is also excited reflexly. There is a prevailing view that the splanchnic circulation must be involved as it is controlled largely from below T5 [36].

Animal experiments have suggested that primary afferent nociceptor inputs (containing calcitonin gene-related peptide but not Substance P) sprout within the cord around the lesion [36], probably due to the release of neurotrophins by inflammatory cells in the segments around the lesion site. However, as mentioned above, sympathetic discharge following brief stimuli in spinally-injured people is not particularly large or prolonged, suggesting that the expansion of afferent connections is not responsible for much enhancement of discharge. Thus the mechanisms underlying the pronounced vasoconstriction during $\mathrm{AD}$ have been unclear.

\section{Changes in vascular reactivity after spinal cord injury}

If the sympathetic discharge after SCI is very much reduced and the reflex discharges provoked by e.g. bladder stimulation are not particularly marked, why does dysreflexia develop? The prolonged vasoconstriction might be explained if neurally evoked vasoconstriction was in some way potentiated. This might result from the potentiated release of transmitter per impulse, as has been shown at ganglionic [9] and central [24] synapses when ongoing activity is blocked. To test this idea, isolated vessel segments were mounted in a myograph and contractile responses evoked by short trains of supramaximal transmural electrical stimuli at $0.1-5 \mathrm{~Hz}$, frequencies within the physiological range. Vessels were isolated from rats with spinal transection at T4 or T8, leaving all parts of the sympathetic pathways intact, and compared with those from age-matched and sham- 
Fig. 2 Contractile responses of segments of (A) cutaneous (tail) artery and (B) splanchnic (mesenteric) artery isolated from (above) control rats and (below) rats 7-8 weeks after spinal transection at (A) T8 and (B) T4. Trains of the same number of transmural electrical stimuli at different frequencies (indicated by bars at bottom) were presented to each vessel to activate the sympathetic postganglionic terminals. Responses in vessels from spinalized rats were greatly enhanced compared with controls. Redrawn from (A) Yeoh et al. (2004a) and (B) Brock et al. (2006)
A

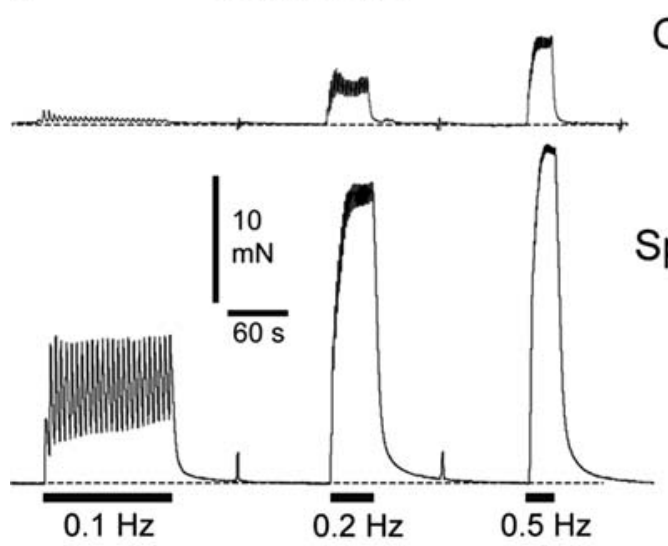

B Splanchnic

Control

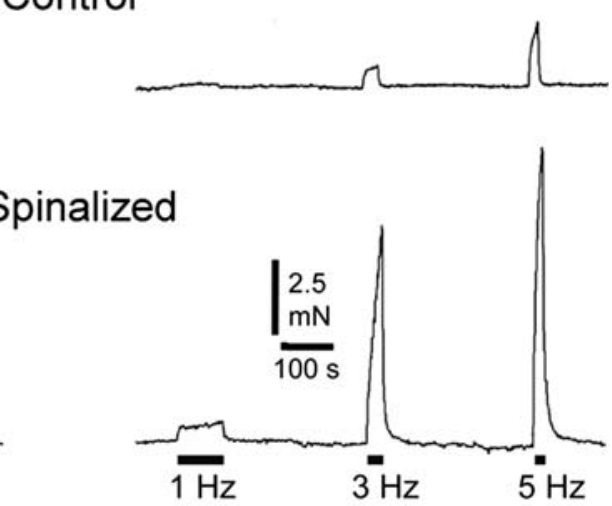

operated controls. After 2 or 8 weeks, the responses of cutaneous and splanchnic arteries to stimulation of the perivascular nerves were greatly enhanced (Fig. 2).

Transmission in small diameter blood vessels is known to occur primarily at neurovascular junctions on the outer layers of smooth muscle cells $[11,18]$. Even when the relation between the postganglionic varicosities and the effector tissue is not direct, it has been shown in all preparations so far investigated that responses to nerve-evoked and to exogenous transmitters do not involve the same receptors or postreceptor mechanisms [11]. The enhanced effect of endogenous neurotransmitters on blood vessels after spinal transection might involve one or more of a number of mechanisms (increased norepinephrine (NE) release, decreased NE clearance, increased junctional receptor sensitivity, increased postreceptor responsiveness), only some of which can be tested directly. Rather unexpectedly, the cellular mechanisms underlying the enhanced responsiveness to $\mathrm{NE}$ released from postganglionic nerve terminals were not the same for each vessel. In the rat tail artery, in which contractile responses to nerve stimulation were enhanced more than 10 -fold, most of the changes in neurovascular transmission were postsynaptic, involving an increased reactivity of the smooth muscle [38]. There was some maintained increase in sensitivity of $\alpha 2$ - but not $\alpha 1$-adrenoceptors to exogenous NE. In contrast, in the mesenteric artery, where transmission was increased 5-fold, most of the changes were presynaptic and involved a decrease in the activity of the NE transporter that takes the released $\mathrm{NE}$ back up into the postganglionic terminals [2]. Differences between the vessels in the ability of $\alpha$ adrenoceptor and purinoceptor antagonists to inhibit nerve-evoked responses imply that the mechanisms of neurovascular transmission are selective for different vascular beds.
To test whether the enhanced responsiveness resulted from the removal of ongoing activity in the postganglionic axons, activity to the tail artery was prevented in normal animals by cutting the lumbar paravertebral chain to disconnect the preganglionic input to the postganglionic neurones (decentralization, Fig. 3(2)). Neurovascular transmission in the tail artery was enhanced after decentralization in the same way as after spinal transection [37]. Notably, sensitivity of the vascular muscle to $\alpha 1$-adrenoceptors was not modified. Even after denervation of the tail artery (by cutting the postganglionic nerves, Fig. 3(3)) to remove the perivascular terminals (and thus the NE transporter), the responses to phenylephrine were much more enhanced relative to control than those to another $\alpha 1$-agonist,

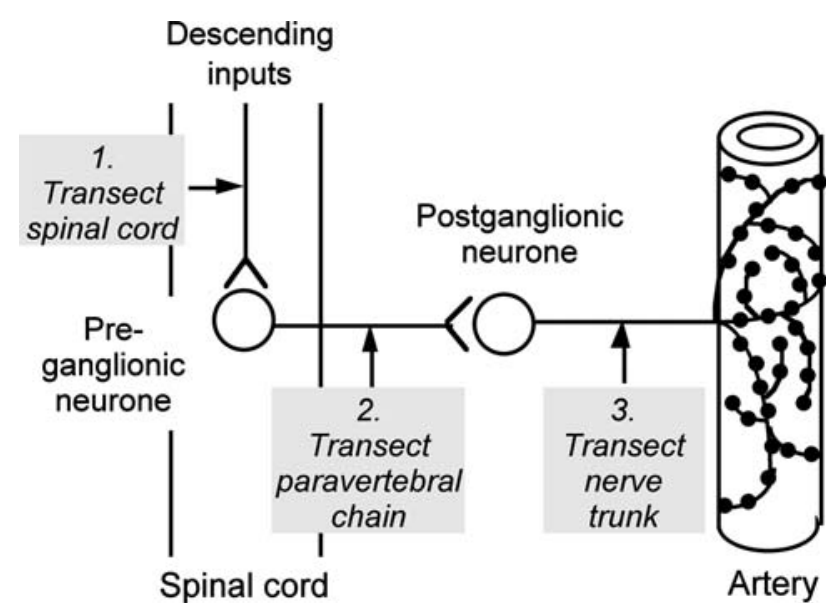

Fig. 3 Diagram showing sites of lesions used to study long term effects on neurovascular transmission in arterial vessels of rats. (1) Transection of thoracic spinal cord without damage to preganglionic neurones. (2) Transection of paravertebral chain to remove preganglionic inputs (decentralization). (3) Transection of postganglionic nerves to denervate artery. Segments of artery were removed from the animals after 2-8 weeks and contractile responses to stimulation of perivascular sympathetic nerves were recorded during exposure to adrenoceptor antagonists and other drugs. that selectively interfere with neurovascular mechanisms 
methoxamine, that is not a substrate for the NE transporter. These observations emphasize the significant role of the postganglionic nerve terminals in reducing the effective concentration of catecholamines applied to the outside of vascular preparations.

Overall, the data so far suggest that blood vessels in spinalized animals would constrict more powerfully to even short bursts of sympathetic activity evoked reflexly below the lesion. As well as being consistent with the complaints of many spinally-injured people about cold feet and legs, and the common difficulties in healing pressure sores, this modified vascular reactivity could contribute strongly to the development of autonomic dysreflexia in humans.

\section{Conclusions}

Despite many years of investigation, we still know remarkably little about the way in which neurallyreleased transmitters, including NE, lead to constriction of vascular smooth muscle. It is now clear that, as for many autonomically-innervated tissues, the mechanisms are not the same as those activated by exogenous transmitters. The work discussed here emphasizes the diversity of mechanisms that underlie the neural control of arterial vessels in different vascular beds and the plasticity of both innervation and effector tissues after lesions to the nervous system. Marked changes can occur even when both pre- and postganglionic components of the sympathetic innervation are undamaged. While the findings in experimental animals need to be confirmed in spinally injured people, they may help us to devise better ways to improve their rehabilitation and long-term maintenance. In addition, we may need to revise our thinking about the role of sympathetic activity in the regulation of the cellular processes involved in neurovascular transmission, even in intact individuals.

Acknowledgements Work in the authors' laboratory was supported by the National Health \& Medical Research Council of Australia and the Christopher Reeve Paralysis Foundation. I am very grateful to James Brock, my collaborator in the experiments on vascular responsiveness after spinal cord injury, for his helpful comments on the manuscript.Disclosure: The author has no financial or other conflict of interest.

\section{References}

1. Anderson CR, McLachlan EM, SrbChristie O (1989) Distribution of sympathetic preganglionic neurons and monoaminergic nerve terminals in the spinal cord of the rat. J Comp Neurol 283:269-284

2. Brock JA, Yeoh M, McLachlan EM (2006) Enhanced neurally evoked responses and inhibition of norepinephrine reuptake in rat mesenteric arteries after spinal transection. Am J Physiol-Heart Circ Physiol 290:H398-405

3. Dampney RA, Coleman MJ, Fontes MA, Hirooka Y, Horiuchi J, Li YW, Polson JW, Potts PD, Tagawa T (2002) Central mechanisms underlying short- and long-term regulation of the cardiovascular system. Clin Exp Pharmacol Physiol 29:261-268

4. Dembowsky K (1995) Integrative properties of sympathetic preganglionic neurones within the thoracic spinal cord. Clin Exp Hypertens 17:313-321

5. Dembowsky K, Czachurski J, Seller H (1985) An intracellular study of the synaptic input to sympathetic preganglionic neurones of the third thoracic segment of the cat. J Auton Nerv Syst 13:201-244
6. Deuchars SA, Milligan CJ, Stornetta RL, Deuchars J (2005) GABAergic neurons in the central region of the spinal cord: a novel substrate for sympathetic inhibition. J Neurosci 25:1063-1070

7. Deuchars SA, Spyer KM, Gilbey MP (1997) Stimulation within the rostral ventrolateral medulla can evoke monosynaptic GABAergic IPSPs in sympathetic preganglionic neurons in vitro. J Neurophysiol 77:229-235

8. Eckberg DL (2000) Physiological basis for human autonomic rhythms. Ann Med 32:341-349

9. Gallego R, Geijo E (1987) Chronic block of the cervical trunk increases synaptic efficacy in the superior and stellate ganglia of the guinea-pig. J Physiol 382:449-462

10. Häbler HJ, Jänig W, Michaelis M (1994) Respiratory modulation in the activity of sympathetic neurones. Prog Neurobiol 43:567-606

11. Hirst GD, Choate JK, Cousins HM, Edwards FR, Klemm MF (1996) Transmission by post-ganglionic axons of the autonomic nervous system: the importance of the specialized neuroeffector junction. Neuroscience 73:7-23
12. Inokuchi H, Yoshimura M, Yamada S, Polosa C, Nishi S (1992) Fast excitatory postsynaptic potentials and the responses to excitant amino acids of sympathetic preganglionic neurons in the slice of the cat spinal cord. Neuroscience 46:657-667

13. Jänig W (1988) Pre- and postganglionic vasoconstrictor neurones: differentiation, types, and discharge properties. Ann Rev Physiol 50:525-539

14. Jänig W (2006) The integrative action of the autonomic nervous system: neurobiology of homeostasis. Cambridge University Press, New York, 480 $\mathrm{pp}$

15. Kümmel H (1983) Activity in sympathetic neurons supplying skin and skeletal muscle in spinal cats. J Auton Nerv Syst 7:319-327

16. Liestol K, Maehlen J, Nja A (1987) Two types of synaptic selectivity and their interrelation during sprouting in the guinea-pig superior cervical ganglion. J Physiol 384:233-245

17. Llewellyn-Smith IJ (2002) GABA in the control of sympathetic preganglionic neurons. Clin Exper Pharmacol Physiol 29:507-513

18. Luff SE (1996) Ultrastructure of sympathetic axons and their structural relationship with vascular smooth muscle. Anat Embryol 193:515-531 
19. Macefield VG, Wallin BG (1999) Respiratory and cardiac modulation of single sympathetic vasoconstrictor and sudomotor neurones to human skin. J Physiol 516:303-314

20. Mathias CJ (2006) Orthostatic hypotension and paroxysmal hypertension in humans with high spinal cord injury. Prog Brain Res 152:231-243

21. McLachlan EM (2003) Transmission of signals through sympathetic gangliamodulation, integration or simply distribution? Acta Physiol Scand 177:227235

22. McLachlan EM, Davies PJ, Häbler H-J, Jamieson J (1997) On-going and reflex synaptic events in rat superior cervical ganglion cells. J Physiol 501:165-182

23. Murray JG, Thompson JW (1957) The occurrence and function of collateral sprouting in the sympathetic nervous system of the cat. J Physiol 135:133-162

24. Murthy VN, Schikorski T, Stevens CF, Zhu Y (2001) Inactivity produces increases in neurotransmitter release and synapse size. Neuron 32:673-682

25. Njå A, Purves D (1977) Specific innervation of guinea-pig superior cervical ganglion cells by preganglionic fibres arising from different levels of the spinal cord. J Physiol 264:565-583
26. Pilowsky PM, Llewellyn-Smith IJ, Minson JB, Arnolda LF, Chalmers JP (1995) Substance P and serotonergic inputs to sympathetic preganglionic neurons. Clin Exper Pharmacol Physiol 17:335-344

27. Purves D, Snider WD, Voyvodic (1988) Trophic regulation of nerve cell morphology and innervation in the autonomic nervous system. Nature 336:123-128

28. Purves D, Wigston DJ (1983) Neural units in the superior cervical ganglion of the guinea-pig. J Physiol 334:169178

29. Sah P, McLachlan EM (1995) Membrane properties and synaptic potentials in rat sympathetic preganglionic neurons studied in horizontal spinal cord slices in vitro. J Auton Nerv Syst 53:1-15

30. Skok VI, Ivanov AY (1983) What is the ongoing activity of sympathetic neurons? J Auton Nerv Syst 7:263-270

31. Stjernberg L, Blumberg H, Wallin BG (1986) Sympathetic activity in man after spinal cord injury. Outflow to muscle below the lesion. Brain 109:695715

32. Taylor EW, Jordan D, Coote JH (1999) Central control of the cardiovascular and respiratory systems and their interactions in vertebrates. Physiol Rev 79:855-916
33. Wallin BG (2006) Regulation of sympathetic nerve traffic to skeletal muscle in resting humans. Clin Auton Res 16:262-269

34. Wallin BG, Donadio V, Karlsson T, Kallio M, Nordin M, Elam M (2003) Arousal increases baroreflex inhibition of muscle sympathetic activity. Acta Physiol Scand 177:291-298

35. Wallin BG, Fagius J (1988) Peripheral sympathetic activity in conscious humans. Ann Rev Physiol 50:565-576

36. Weaver LC, Marsh DR, Gris D, Brown A, Dekaban GA (2006) Autonomic dysreflexia after spinal cord injury: central mechanisms and strategies for prevention. Prog Brain Res 152:245263

37. Yeoh M, McLachlan EM, Brock JA (2004) Chronic decentralization potentiates neruovascular transmission in the isolated rat tail artery, mimicking the effects of spinal transection. J Physiol 561:583-596

38. Yeoh M, McLachlan EM, Brock JA (2004) Tail arteries from chronically spinalized rats have potentiated responses to nerve stimulation in vitro. J Physiol 556:545-555 